\title{
Fuzzy Control Design for Hypersonic Vehicle
}

\author{
Guo Li ${ }^{1, a}$, Xiaoliang Lv ${ }^{2, b}$ \\ ${ }^{1}$ Automation School, University of Science and Technology Beijing, Beijing, 100083, China \\ ${ }^{2}$ Automation School, University of Science and Technology Beijing, Beijing, 100083, China \\ aguo6393@sina.com, blv_maxwell@sina.com
}

Keywords: Hypersonic Vehicle; T-S Model; PDC; LMI.

\begin{abstract}
In this paper, firstly, the non-linear longitudinal modeling approach is proposed based on the geometric shape of the typical structure of the hypersonic vehicle as the research object, combined with correlative hypersonic aerodynamic theories, and then local linear models are established near multiple balance points of the overall fuzzy system by utilizing fuzzy Takagi-Sugeno (T-S) modeling based on different areas dynamic characters. An overall non-linear fuzzy model is obtained by the integration of these local models linking fuzzy membership function. Parallel distributed compensation (PDC) control algorithm is proposed based on built T-S fuzzy model by considering the linear state feedback controller for each of the fuzzy local model. Moreover, sufficient condition for the stability of T-S fuzzy system is investigated for the non-linear system which has complex stability analysis by obtaining a common positive definite matrix $P$ that would satisfy a set of Lyapunov inequalities and the state feedback controller gain matrixes $\mathrm{K}$ based on linear matrix inequalities (LMI) optimization algorithm. Finally, MATLAB is utilized to realize result simulation to show the effectiveness of the proposed technique, including performance quality and robustness requirements.
\end{abstract}

\section{Introduction}

Hypersonic aircraft is a highly nonlinear multivariable system, due to the adoption of advanced design integrating airframe and engine, the supersonic combustion ramjet and structure of large slenderness ratio, makes it to own strong coupling, high-dynamic pressure elastic deformation and parameter time-varying characteristics in a wide range [1]. Such as flying at a high pressure condition, the control efficiency compared with subsonic and supersonic flight state has greatly reduced, the influence of external disturbance of hypersonic vehicle is very significant. Therefore, hypersonic aircraft flight control system should be to have stronger robustness to deal with outside interference and other uncertain factors [2][3].

Take hypersonic vehicle of Hyper-X series [4] which NASA has developed as the background, establish T-S model of fuzzy controller. Parallel distributed compensation (PDC) control algorithm is proposed based on built T-S fuzzy model. Finally, it was studied about reasonable and effective simulation control for hypersonic vehicle.

\section{Dynamic Modeling and Analysis of Hypersonic Vehicle}

The Longitudinal Dynamics Model. This article mainly research open hypersonic vehicle longitudinal motion of NASA Langley laboratory [5][6].

In the course of modeling, assumes the engine thrust direction along the axis of engine, and parallel to the axis of fuselage; Used thrust coefficient $C_{T}$ (or the opening of the throttle valve $\beta$ ), the elevator Angle $\delta_{e}$ for input, and selected $x=\left[\begin{array}{lllll}V & \gamma & h & \alpha & q\end{array}\right]$ as state variables respectively for hypersonic vehicle speed, track angle, altitude, angle of incidence and pitching angle velocity. According to the stress distribution, nonlinear equations of high velocity aircraft longitudinal dynamic model can be described as follow on the speed of coordinate system:

$$
\dot{V}=(T \cos \alpha-D) / m-(\mu \sin \gamma) / \gamma^{2}
$$




$$
\begin{aligned}
& \dot{\gamma}=(L+T \sin \alpha) / m V-\left[\left(\mu-V^{2} \gamma\right) \cos \gamma\right] / V \gamma^{2} \\
& \dot{h}=V \sin \gamma \\
& \dot{\alpha}=q-\dot{\gamma} \\
& \dot{q}=M_{y y} / I_{y y}
\end{aligned}
$$

In the above formula, each variable values refer to [7], Engine dynamics model can be described to a second order system:

$$
\ddot{\beta}=-2 \xi \omega_{n} \dot{\beta}-\omega_{n}^{2} \beta+\omega_{n}^{2} \beta_{c}
$$

Local Model Linearization Analysis. Cruise equilibrium operating point state for hypersonic vehicle is $V_{0}=4590.3, h_{0}=33528, \gamma_{0}=0, q_{0}=0, \alpha_{0}=0.10314$, It's corresponding input balance point is $\delta_{e}=-0.00695, C_{T}=4.5338 \times 10^{-3}$. According to the general linear theory, we can find the corresponding matrix:

$$
\begin{aligned}
& A=\left[\begin{array}{lllll}
-465.5112 \times 10^{-6} & -9.7042 & 72.8030 \times 10^{-6} & -13.5298 \times 10^{-6} & 0 \\
893.2801 \times 10^{-9} & 0 & -163.9731 \times 10^{-9} & 13.3085 \times 10^{-3} & 0 \\
0 & 45900 \times 10^{-3} & 0 & 0 & 0 \\
-893.2801 \times 10^{-9} & 0 & 163.9731 \times 10^{-9} & -13.3085 \times 10^{-3} & 1 \\
1.8121 \times 10^{-6} & 0 & -129.2264 \times 10^{-9} & 2.0807 \times 10^{-3} & -24.8355 \times 10^{-3}
\end{array}\right] \\
& B=\left[\begin{array}{ll}
97.9551 & 0 \\
10.1391 & 0 \\
0 & 0 \\
-10.1391 & 0 \\
0 & 308.0969 \times 10^{-3}
\end{array}\right]
\end{aligned}
$$

According to the matrix A, the characteristic root of the linear model of the system is calculated: $\lambda_{1,2}=-49.7536 \times 10^{-3} \pm 20.5992 \times 10^{-3} i, \lambda_{3,4}=30.9712 \times 10^{-3} \pm 35.8092 \times 10^{-3} i$,

$\lambda_{5}=-1.0449 \times 10^{-3}$

It can be seen that the complex roots close to the imaginary axis, system close to critical stable, it is easy to produce shock, at the same time, there are positive real roots to make the system unstable. So we need to add the controller to make the system stable.

\section{The Analysis and Design of the Hypersonic Vehicle T-S Model}

Simplified Mathematical Model of Hypersonic Vehicle. In order to acquire simply controller, take the method of following high with fixed speed or speed with constant high, the original five order nonlinear system model is simplified as a three or four order nonlinear system model under simple processing. When set $h \equiv 33528$, it is easy to obtain $\gamma \equiv 0$. Then the original nonlinear model is changed into three order systems as following:

$$
\dot{V}=(T \cos \alpha-D) / m
$$


$\dot{\alpha}=q$

$\dot{q}=M_{y y} / I_{y y}$

Among them, the formula Eq.7-Eq.9 satisfies the following constraints:

$(L+T \sin \alpha) / m V=\left(\mu-V^{2} \gamma\right) / V \gamma^{2}$

Hypersonic Vehicle T-S Modeling. Based on local linearization theory and T-S model analysis [8], the dynamic equation of hypersonic vehicle can be classified by the $(V, \alpha, q)$ plane, and the local linearization of hypersonic vehicle is realized.

Hypersonic vehicle model described by T-S fuzzy rules:

$\dot{x}(t)=\sum_{i=1}^{12} \mu_{i}(z(t))\left[A_{i} x(t)+B_{i} u(t)\right]$

\section{Analysis and Design of Fuzzy Controller for Hypersonic Vehicle}

The design of state feedback controller is studied by using the method of constraining control input. By $\left(A_{i}, B_{i}\right)$, we know that system is locally controlled, and obtain matrix $\mathrm{Kn}$ of the state feedback controller and the the common positive definite matrix $\mathrm{P}$ satisfying Lyapunov inequality by solving the LMI inequality [9], then the state feedback controller is designed:

$$
u(t)=-\sum_{i=1}^{12} \mu_{i}(z(t)) K_{i} x(t)
$$

In summary, a hypersonic vehicle model described in the form of 12 T-S fuzzy rules can be obtained:

$$
\dot{x}(t)=\sum_{i=1}^{12} \sum_{j=1}^{12} \mu_{i}(z(t)) \mu_{j}(z(t))\left[A_{i} x(t)+B_{i} K_{j}\right] x(t)
$$

At this point, the Eq.7-Eq.9 which describes the hypersonic vehicle longitudinal nonlinear model following speed with fixed high were transformed into a closed-loop system with state feedback controller Eq.13.

\section{Analysis and design of a hypersonic vehicle tracking controller}

Hypersonic vehicle model with T-S fuzzy rules:

$$
\dot{\hat{x}}(t)=\sum_{i=1}^{12} \mu_{i}(z(t))\left[\hat{A}_{i} \hat{x}(t)+\hat{B}_{i} u(t)\right]+\left[\begin{array}{l}
d_{r} \\
y_{r}
\end{array}\right]
$$

Similar to the design of a hypersonic vehicle PDC controller, a state feedback controller [10] is designed:

$$
u(t)=-\sum_{i=1}^{12} \mu_{i}(z(t)) \hat{K}_{i} \hat{x}(t)
$$

In summary, the formula Eq.15 is substituted into the formula Eq.14, the overall model described with 12 T-S fuzzy rules of the superb hypersonic vehicle tracking control was obtained:

$$
\dot{x}(t)=\sum_{i=1}^{12} \sum_{j=1}^{12} \mu_{i}(z(t)) \mu_{j}(z(t))\left[\hat{A}_{i} \hat{x}(t)+\hat{B}_{i} \hat{K}_{j}\right] \hat{x}(t)+\left[\begin{array}{l}
d_{r} \\
y_{r}
\end{array}\right]
$$




\section{Simulation}

Control Simulation of Hypersonic Vehicle. For the design of PDC controller, the simulation results were presented based on following the high and constraining speed. Where initial conditions is $\left(V_{0}=4000, \alpha_{0}=0, q_{0}=0.26\right)$.

In Fig. 1, the simulation results show that the final velocity $(V)$ is stable at $4600 \mathrm{~m} \cdot \mathrm{s}^{-1}$, the angle of attack is near $0.10083 \mathrm{rad}$, the slope angle is stable at 0 , and the thrust coefficient $\left(C_{T}\right)$ is near 0.013703. Compared with the control output of the high speed vehicle at $V_{\infty}=4590 \mathrm{~m} \cdot \mathrm{s}^{-1}$, the control output of the system has some errors, so it needs to design the controller to eliminate the steady-state error of the system.

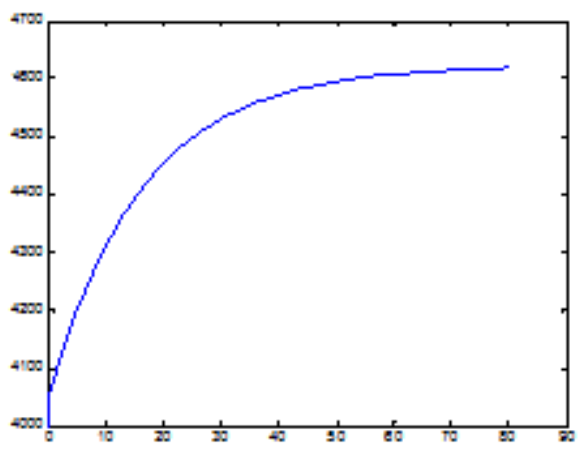

(a) The speed

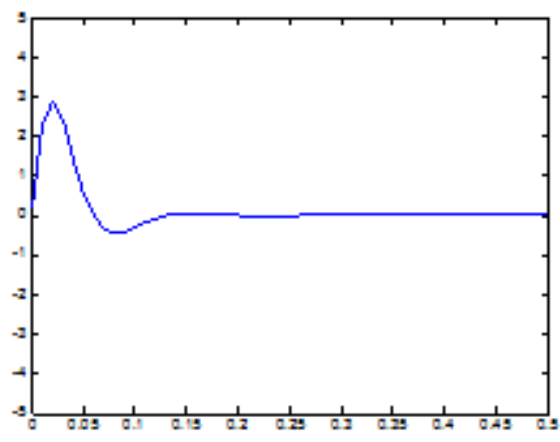

(c) Inclined angle velocity

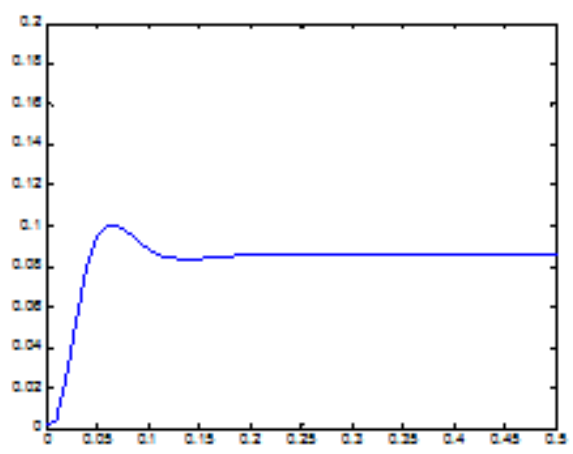

(b) Angle of attack

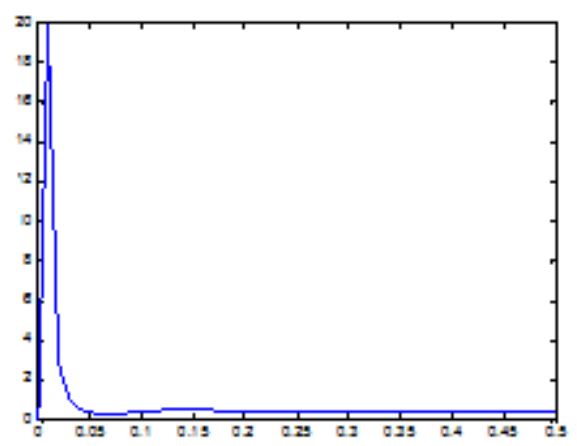

(d) Thrust coefficient control input

Fig. 1 The experimental results

Tracking Control Simulation of Hypersonic Vehicle. For the PDC tracking controller, the simulation results of hypersonic vehicle were given based on following the high and constraining speed. The initial conditions are $V_{0}=0, \alpha_{0}=0, q_{0}=0$ [30].

(1) In Fig. 2, given speed is $y_{r}=5500$, and when the interference signal $d_{r}=\left[\begin{array}{lll}25 & 0.008 & 0.06\end{array}\right]$ is applied, the duration is $0.2 \mathrm{~s}$ :

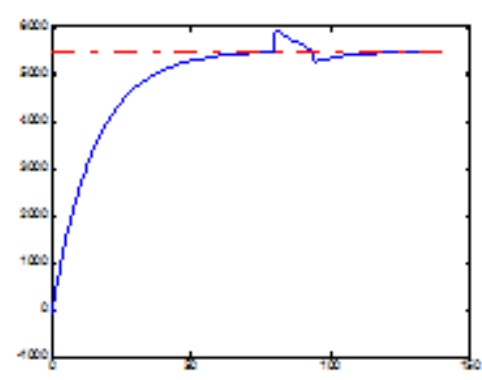

(a) The speed

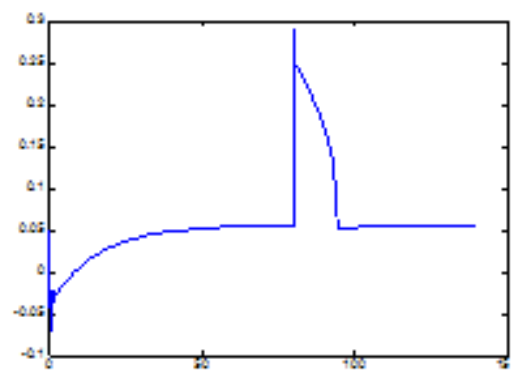

(b) Angle of attack

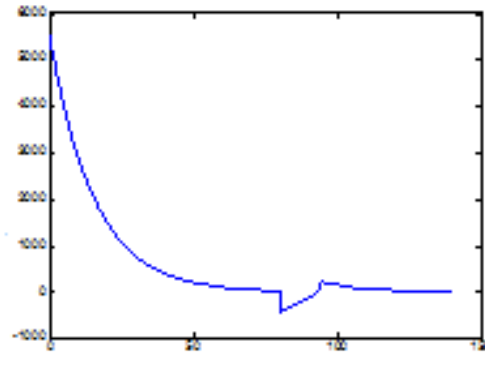

(c) Inclined angle velocity 


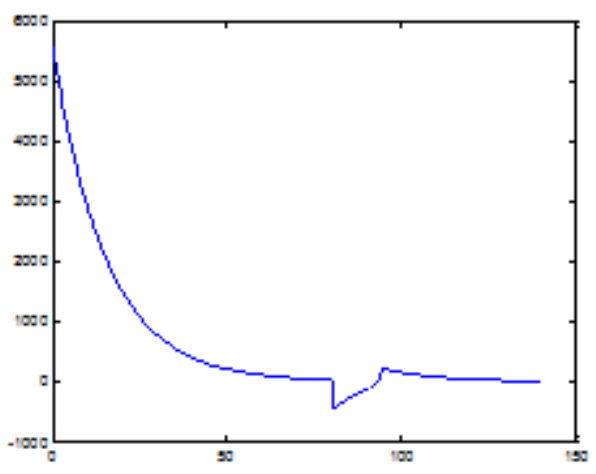

(d) speed error

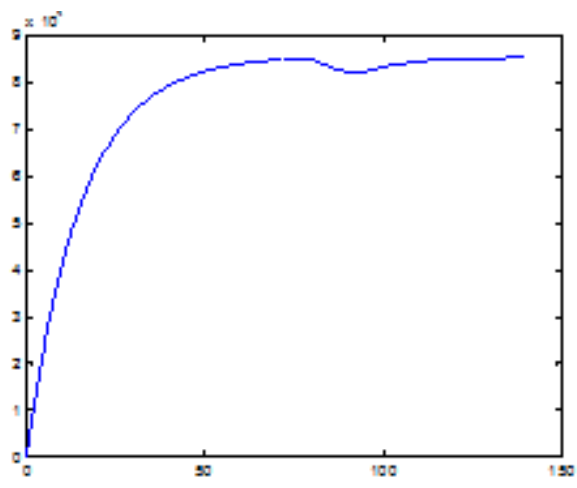

(e) Error integral curve

Fig.2 Given point curve

(2) In Fig.3, set a given curve $y_{r}=1-e^{-t / 10}$ (as shown in Fig.3 (a)), when the time is $t=40 \mathrm{~s}$, applied interference signal $d_{r}=\left[\begin{array}{lll}25 & 0.008 & 0.06\end{array}\right]$ to system continued 0.2s:

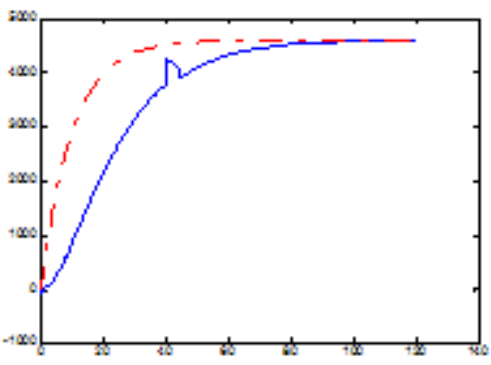

(a) The speed

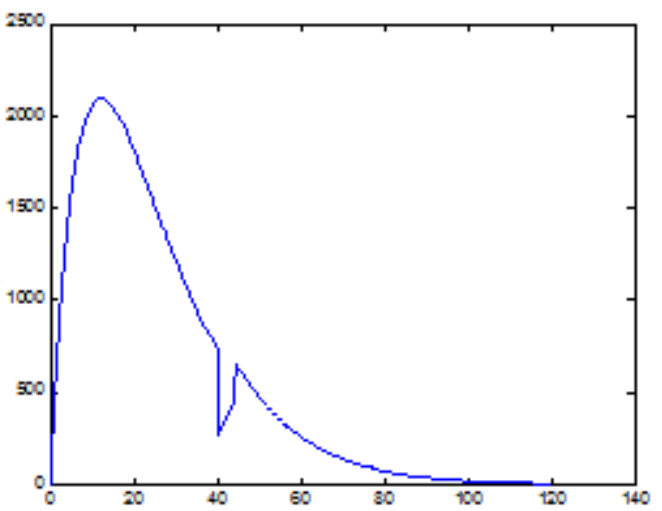

(d) speed error

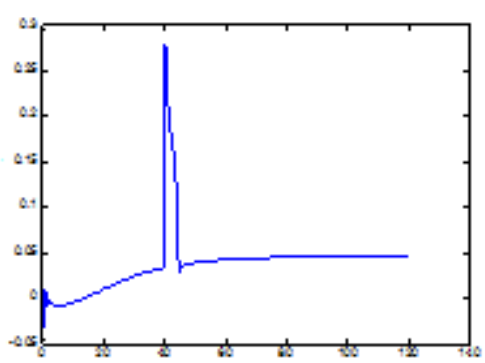

(b) Angle of attack

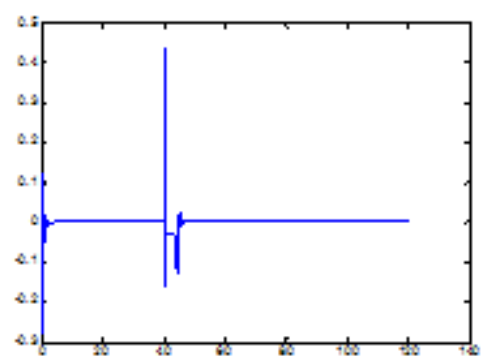

(c) Inclined angle velocity

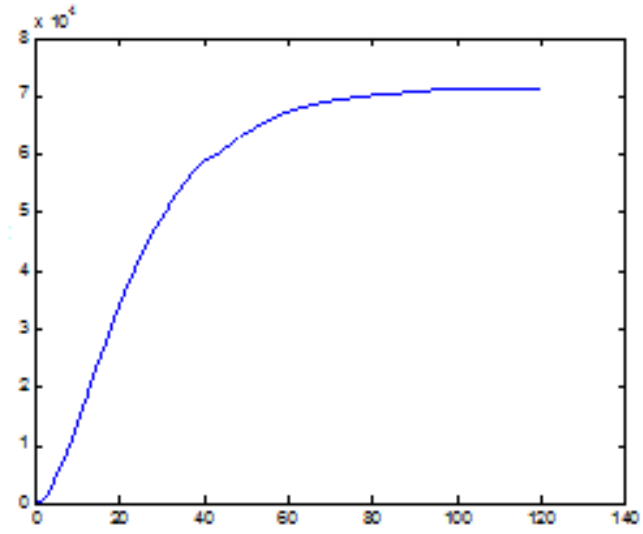

(e) Error integral curve

Fig.3 Given curve

By the above simulation analysis, the hypersonic vehicle can be tracked in a given value or given curve, and the internal state of the system can guarantee the stability of the Lyapunov, and the output will converge to the given input. If there is an external disturbance, the system can return to the steady state in a short time, and the tracking error tends to zero and the system state converges to the equilibrium state.

\section{Conclusion}

In view of the high nonlinearity of hypersonic vehicle and the longitudinal model containing 5 order state variables, we established T-S model, proposed parallel distributed compensation (PDC) control algorithm. First, the T-S model control design method can greatly reduce the number of rules used in control process, always only need establish local linear model in a specific number operating points of the system, control effectiveness also can be improved. Secondly, it is used to analyze the stability of the control system that the former is fuzzy language description, and the latter is exact linear equation for each rule of the T-S model. 


\section{Acknowledgements}

In this paper, the research was sponsored by the Fundamental Research Funds for the Central Universities (Project No. 06105054).

\section{References}

[1] Baris Fidan, Maj.Mirmirani, Petros Aloannous. Flight dynamics and control of air-breaking hypersonic vehicles-Review and new directions[J]. 12th AIAA intenational space planes, 2003, 7081:1-24.

[2] Michael, Bolender, David B, Doman. A non-linear model for the longitudinal Dynamics of a Hypersonic Air-breaking Vehicle[J]. AIAA, 2005-6255.

[3] Heiser W H, Pratt D T. Hypersonic air-breaking propulsion[J]. Washington DC: AIAA, 1994.

[4] Shaughnessy J D, Pinckney S Z, McMinn J D, et al. Hypersonic vehicle simulation model: winged-cone configuration[J]. NASA TM-102610, 1990.

[5] Wu C Gregory M, Chowdhry R S, McMinn J D, et al. Hypersonic vehicle model and control law development using $H_{\infty}$ and $\mu$ synthesis[J]. NASA TM-4562, 1994.

[6] Chavez F R, Schmidt D K. An integrated analytical aero- propulsive/aeroelastic for dynamic analysis of hypersonic vehicles[J]. NASA ARC 92-2, 1992.

[7] Haojian Xu, Maj D. Mirmirani, Petros A. Ioannou. Adaptive Sliding Mode Control Design for a Hypersonic Flight Vehicle[J]. Journal of Guidance, Control, and Dynamics, 2004, 27(5): 829-838.

[8] Marcelo C. M. Teixeira, Stanislaw H. Zak. Stabilizing Controller Design for Uncertain Nonlinear Systems Using Fuzzy Models[J]. IEEE Transactions on Fuzzy Systems, 1999, 7(2): 133-142.

[9] H. D. Tuan, P. Apkarian, T. Narikiyo, Y. Yamamoto. Parameterized linear matrix inequality techniques in fuzzy control system design[J]. IEEE Transactions on Fuzzy Systems, 2001, 9(2): 324-332. 